Revue bibliographique pour le domaine irano-aryen

\title{
Richard K. Larson, Sedigheh Moradi, Vida Samiian (eds.). Advances in Iranian Linguistics
}

\section{Pegah Faghiri}

\section{(2) OpenEdition}

1 Journals

\section{Édition électronique}

URL : https://journals.openedition.org/abstractairanica/53082

DOI : 10.4000/abstractairanica.53082

ISSN : 1961-960X

Éditeur :

CNRS (UMR 7528 Mondes iraniens et indiens), Éditions de l'IFRI

\section{Référence électronique}

Pegah Faghiri, « Richard K. Larson, Sedigheh Moradi, Vida Samiian (eds.). Advances in Iranian Linguistics », Abstracta Iranica [En ligne], Volume 42-43 | 2021, document 7, mis en ligne le 30 décembre 2021, consulté le 13 décembre 2022. URL : http://journals.openedition.org/ abstractairanica/53082 ; DOI : https://doi.org/10.4000/abstractairanica.53082

Ce document a été généré automatiquement le 13 décembre 2022.

Tous droits réservés 


\title{
Richard K. Larson, Sedigheh Moradi, Vida Samiian (eds.). Advances in Iranian Linguistics
}

\author{
Pegah Faghiri
}

\section{RÉFÉRENCE}

Richard K. Larson, Sedigheh Moradi, Vida Samiian (eds.). Advances in Iranian Linguistics. Amsterdam: John Benjamins, (Current Issues in Linguistic Theory 351), 2020, vi+309 p., ISBN: 9789027207166

1 Cet ouvrage regroupe une sélection d'articles, à partir de plus de quarante contributions au programme du colloque 1st North American Conference in Iranian Linguistics (NACLI 1) à Stony Brook en avril 2017. Il s'agit du troisième ouvrage regroupant des travaux en linguistique iranienne, à la suite de Aspects of Iranian linguistics (Karmi, Samiian et Stilo 2009) et Topics in Iraninan linguistics (Korn, Haig, Karimi et Samvelian 2011). En effet, les ouvrages collectifs consacrés à la linguistique iranienne sont bien rares, malgré le potentiel important que les langues iraniennes offrent aux chercheurs en linguistique théorique et en typologie, comme le démontre Sedigheh Moradi dans son article d'introduction. Moradi y décrit cette famille comme un hub stable de diversité en mettant l'accent sur la diversité de ses traits linguistiques tout en préservant une certaine stabilité en dépit des changements depuis des siècles. Cependant, bien que les contributions du colloque incluaient un vaste éventail de langues iraniennes, la majorité des articles réunis dans ce volume porte sur le persan. Dans l'introduction, Moradi justifie cela en se référant à la notion de typologie canonique de Corbett (2003), évoquant la place particulière qu'occupe le persan au sein des langues iraniennes qui rendrait son étude essentielle pour l'établissement d'un cadre permettant de saisir l'éventail des possibilités et de mesurer les degrés de diversité dans les langues iraniennes. En outre, pour permettre de situer les douze chapitres constitutifs du volume et de saisir leurs intérêts et leurs apports, Moradi 
propose une présentation des langues iraniennes ainsi qu'un aperçu des travaux fondamentaux ayant jalonné le domaine de la linguistique iranienne - allant des travaux datant des années 1950 sur le vieux perse jusqu'aux récents travaux de terrain sur les langues régionales telles que le baloutchi ou le vafsi. Notons que la présentation des chapitres se fait dans l'ordre alphabétique et qu'il n'y a pas de groupement thématique.

2 Parmi les douze articles, huit se focalisent sur le persan (chap. 2, 4, 6, 7, 9, 10, 11, 12) sachant que certains évoquent également les données d'autres langues moins étudiées telles que kurmanji, gilaki, sorani, vafsi ou zazaki - et un sur le tat (chap. 13). Quatre articles portent sur des aspects bien connus tels que la construction ezafe ou l'enclitique $r \bar{a}$ en proposant une analyse nouvelle ou un éclaircissement relatif à de nouvelles données. Richard K. Larson et Vida Samiian (chap. 10) proposent une analyse syntaxique de l'ezafe dans le cadre de la théorie de cas; Simin Karimi et Ryan Walter Smith (chap. 9) reviennent sur rā pour proposer une analyse formelle qui rendrait compte de ses différentes fonctions ; Masoud Jasbi (chap. 7) revisite la sémantique de rā en le définissant comme marqueur de présupposition existentielle, et enfin Murad Suleymanov (chap. 13) étudie ra en tat dans un contexte de contact de langues sur la base des données de corpus oral et écrit. Cinq articles se focalisent sur des aspects moins connus et/ou peu étudiés: Jila Ghomeshi (chap.4) étudie les propriétés de (h)am, marqueur d'ajout (additive marker) qu'elle propose d'analyser comme un particule discursif ; Masoud Jasbi (chap. 6) se penche sur -e, le suffixe de définitude du persan familier qui s'attache aussi bien aux noms définis qu'aux indéfinis, tâchant de donner une définition unique de sa fonction; Vahideh Rasekhi (chap. 12) présente deux constructions stripping (dit aussi ellipse polaire, ex. Paul rit et Luc aussi) avec négation et argue que ce sont des propositions coordonnées et obtenues par l'ellipse du TP ; Elias Abdollahnejad et Dennis Ryan Storoshenko (chap.2) étudient les mécanismes de résolution d'anaphore en comparant les propriétés de liage des pronoms personnels un et $x o d=e \check{s}$; Mohsen Mahdavi Mazdeh (chap. 11) étudie le système métrique des chansons folkloriques et populaires iraniennes et argue qu'elles suivent les mêmes principes que la poésie classique persane. Enfin, le volume comprend trois articles portant sur des langues iraniennes dans leur ensemble (chap. 3,5,8): Erik Anonby, Amos Hayes et Robert Oikle (chap. 3) proposent une nouvelle approche multidimensionnelle pour la classification des langues et dialectes mise en œuvre dans Atlas of the Languages of Iran (ALI) ; Geoffrey Haig (chap. 5) étudie la grammaticalisation des marqueurs d'accord en se penchant sur l'asymétrie entre le sujet et l'objet, et Thomas Jügel et Pollet Samvelian (chap. 8) proposent une étude diachronique des pronoms personnels clitiques.

\section{Table des matières}

Chapter 1. Advances in Iranian linguistics: An introduction. Sedigheh Moradi (p. 2-13)

Chapter 2. Syntactic and semantic constraints on pronoun and anaphor resolution in Persian. Elias Abdollahnejad and Dennis Ryan Storoshenko (p.15-27)

Chapter 3. A multi-dimensional approach to classification of Iran's languages. Erik Anonby, Amos Hayes and Robert Oikle (p. 29-56)

Chapter 4. The additive particle in Persian: A case of morphological homophony between syntax and pragmatics. Jila Ghomeshi (p. 57-83)

Chapter 5. The pronoun-to-agreement cycle in Iranian: Subjects do, objects don't. Geoffrey L.J. Haig (p. 85-106) 

is. Masoud Jasbi (p. 119-135) Chapter 8. Topic agreement, experiencer constructions, and the weight of clitics. Thomas Jügel and Pollet Samvelian (p. 137-153) (cf. le compte rendu de l'article dans ce même numéro d'AbstIr)

Chapter 9. Another look at Persian rā : A single formal analysis of a multi-functional morpheme. Simin Karimi and Ryan Walter Smith (p. 155-172)

\section{6)}

14 Mazdeh (p. 237-255)

Chapter 13. Oblique marking and adpositional constructions in Tat: A mosaic of dialectal convergence and divergence. Murad Suleymanov (p. 275-300)

Author index (p. 301-304)

Languages index (p. 305-306)

Subject index (p. 307-309)

\section{AUTEURS}

\section{PEGAH FAGHIRI}

University of Amsterdam 